\title{
APTASENSORS FOR EMERGING ENVIRONMENTAL POLLUTANTS
}

\author{
Gaelle Catanante ${ }^{1}$, Atul Sharma ${ }^{1,2}$, Akhtar Hayat $^{1,3}$, Jean Louis Marty ${ }^{1}$ \\ ${ }^{1}$ BAE Laboratory, UPVD, 52 Avenue Paul Alduy, Perpignan 66860, France \\ 2 Biosensor Lab, Department of Chemistry, BITS, Pilani-K. K. Birla Goa Campus, Zuarinagar, \\ Goa 403726, India \\ ${ }^{3}$ IRCBM, COMSATS Institute of Information Technology (CIIT), Lahore 54000, Pakistan
}

\begin{abstract}
With the increasing incidences of hazards to human health, agriculture and ecosystems, the regular monitoring of environmental contaminants such as toxins, pathogens, pharmaceuticals, resulting in various environmental borne diseases are highly recommended. As an example, according to World Health Organization (WHO), the increased mortality of food borne and waterborne diarrheal diseases resulting in 2.2 million deaths per annum, out of which mostly (1.9 million) are children's. In the recent years, the stubbornly increasing public health problems and food contamination of environmental pollutants has increased the demands of designing and fabricating the more reliable and field suitable technologies for cost effective and on-site analysis. Recently, the advances in the nucleic acid research led to the identification of specific oligonucleotides acid sequences, which are highly specific to their cognate targets and known as "aptamers". Aptamers as synthetic bio-recognition element can be in-vivo generated against a variety of analytes such as organic ions/protein/macromolecules with dissociation constants typically varying from the micro to pico-molar range. Recently, the aptamer have been widely exploited to develop the aptamerbased biosensors, commonly known as aptasensors. Aptasensors represents the novelty in the environmental and analytical science with their promising performance of high selectivity, sensitivity and stability over conventional analytical tools. In the present work, the aptasensors presented has focused on the recent advances in the development of aptamer based electrochemical or optical sensors for environmental applications with special emphasis on emerging pollutants described in the literature or develop in our laboratory BAE.
\end{abstract}

Keywords: antibiotic, aptasensor, biotoxin, mycotoxin, pathogen

\section{Introduction}

The occurrence of environmental contamination, especially mycotoxin and antimicrobial residue in food, agriculture and environment, due to their potential threat to human health and environment has drawn significant attention worldwide. Mycotoxins are the secondary toxic metabolite of fungal species commonly molds i.e. Aspergillus, Penicillium and Fusarium [1]. In recent years, the presence of mycotoxins (aflatoxins, ochratoxin) in food, feed and beverages due to their toxicological side effects on the human health and the environment now has become a significantly primary concern [2]. Concerning to the incidences of "suprainfection" caused due to the secondary effects of antibiotics and increased microbial resistance, ensuring the use of suitable amounts of antibiotics in clinical, veterinary and agriculture industry is probably the major health problem [3, 4]. According to the World Health Organization (WHO), the increased incidences and mortality of food and waterborne diarrheal diseases resulting in 2.2 million deaths/annum, among them mostly (1.9 million) are children's. To insure the quality of food, human health and environment and safety to human health, the European Commission (EC) have established the maximum residue 
limits (MRLs) for most of the mycotoxins in food, beverages and food products based on their methods of sampling and analysis for control $[5,6]$. Various stringent limits have already mandated for the presence of antimicrobial residues in different food matrices and environmental samples by the regulatory agencies such as United States Food and Drug Administration (USFDA), Food Safety and Standard Authorities of India (FSSAI), Codex Alimentarious Commission (CAC) and European Union (EU) [7-9]. Therefore, it is highly critical to design and develops highly sensitive and selective analytical methods for regular monitoring and routine analysis of environmental contaminants (antimicrobial and mycotoxins) in food, clinical diagnosis and environment. Over the years, several conventional and immunoassay based techniques have been reported for detection of these contaminants but the inherent properties involving the lengthy process control, animal immunization, large sample and time consuming process involvement limits their utility. Recently, the emergence of biosensors especially the electrochemical aptasensor have gained a significant attention due to advantages of high sensitivity, selectivity, low detection, portability and on-site analysis. In the present work, we are focusing on the aptasensors for detection of antimicrobial and mycotoxins in various food matrices developed in our lab (Laboratory BAE).

\section{Experimental}

a. Chemicals and materials

i. Mycotoxins (Aflatoxin and ochratoxin)

Amino modified aptamers of AFB1 and the anti-OTA aptamer tagged with redox probe methylene blue (MB) were purchased from M/s Microsynth (Schutzenstrasse, Balgach, Switzerland). The aptamer sequences are shown below.

- (5'-NH2 modified) 5'-

TGGGGTTTTGGTGGCGGGTGGTGTACGGGCGAGGG-3. (AFB1-NH2)

- (5'- COOH and 3'-MB modified) 5'-

GATCGGGTCTGGGTGGCGTAAAGGGAGCA TCGGACA-3' (COOH-OTA-MB).

All other chemicals, dibasic sodium phosphate, monobasic potassium phosphate, sodium hydrogen carbonate, magnesium chloride, potassium chloride, sulfuric acid $(98 \%)$, acetonitrile $(98 \%)$, sodium chloride, ethanol $(98 \%)$, tetrabutylammonium tetrafluoroborate $99 \%\left(\mathrm{NBu}_{4} \mathrm{BF}_{4}\right)$, sodium nitrite, N-hydroxysuccinimide (NHS), N-(3dimethylaminopropyl)-N-ethyl-carbodiimide hydrochloride (EDC), potassium ferrocyanide $\left(\mathrm{K}_{4}\left[\mathrm{Fe}(\mathrm{CN})_{6}\right]^{4-}\right)$, potassium ferricyanide $\left(\mathrm{K}_{3}\left[\mathrm{Fe}(\mathrm{CN})_{6}\right]^{3-}\right)$, 4-aminobenzoic acid, hydrochloric acid, ethanolamine, hexamethylenediamine (HMDA), poly(ethylene glycol) diamine $\mathrm{NH}_{2}-\mathrm{PEG}-\mathrm{NH}_{2}(\mathrm{Mw} 3000$ and 10,000), and bovine serum albumin were purchased from Sigma (France). All reagents were of analytical grade. Aflatoxin B1 purchased from M/s Sigma Aldrich (St. Quentin Fallavier Cedex, France) was firstly dissolved in 1:1 v/v methanol: chloroform mixture at $0.5 \mathrm{mg} \cdot \mathrm{mL}^{-1}$ concentrations, and then further dilutions were prepared in BB. For practical application, the beer and wine samples were purchased from a local supermarket at Perpignan, France. Ochratoxin A (from A.ochraceous) and Ochratoxin B (OTB) procured from Sigma was first dissolved in methanol and then diluted in BB. Aptamer solutions were prepared in binding buffer (BB, pH 7.4) containing $0.1 \mathrm{mM} \mathrm{Na}_{2} \mathrm{HPO}_{4}$ and $1.8 \mathrm{mM} \mathrm{KH}_{2} \mathrm{PO}_{4}, 1 \mathrm{mM}$ $\mathrm{MgCl}_{2}, 140 \mathrm{mM} \mathrm{NaCl}$ and $2.7 \mathrm{mM} \mathrm{KCl}$

ii. Antimicrobial residue (Kanamycin) 
The sequence of amino modified anti KANA-aptamer synthesized and purified from $\mathrm{M} / \mathrm{s}$ Microsynth (Switzerland) was used. Aptamer sequence is shown as: (3'- $\mathrm{NH}_{2}$ modified) 5'-TGGGGGTTGAGGCTAAGCCGA-3'-NH2. Kanamycin sulphate was procured from Sigma Aldrich (France) used were analytical grades. All the other chemicals and solvents were used as received. Milk sample was purchased from the local market in Perpignan, France. A $10 \mathrm{mM}$ binding buffer ( $\mathrm{pH}$ 7.4) was used for aptamer preparation.

\section{b. Apparatus}

All the electrochemical measurements were performed on an Autolab PGSTAT100 potentiostat/galvanostat equipped with a frequency response analyzer (FRA) system (Eco Chimie, Netherlands) controlled by the two Autolab softwares i.e. Frequency Response analyzer for impedance and General purpose Electrochemical system for voltammetry with version 4.9. Screen printed carbon electrodes (SPCEs) with a customized designed of conventional three electrodes system were fabricated using a DEK 248 screen-printing system (BAE Laboratory, Perpignan, France) [10]. The impedance spectra were recorded using a sinusoidal ac potential perturbation of $5 \mathrm{mV}$ in the frequency range $10^{3}-0.1 \mathrm{~Hz}$ with 20 discrete frequencies per decade, superimposed on a dc potential of $0.1 \mathrm{~V}$. All impedimetric measurements were performed in a solution of $1.0 \mathrm{mM}\left[\mathrm{Fe}(\mathrm{CN})_{6}\right]^{4-/ 3-}$ in $\mathrm{BB} \mathrm{pH} 7.4$ as a background electrolyte. Surface characterization was performed using IR Affinity-1 (SHIMADZU, Japan) attached with attenuated total reflectance (ATR) attachment.

\section{i. Covalent aptamer immobilization via diazonium functionalized SPCE}

Prior to use, surface modification, the SPCE was subjected to electrochemical pretreatment by applying several potential cycles from 1.0 to $-1.5 \mathrm{~V}$ vs. pseudo $\mathrm{Ag} / \mathrm{AgCl}$ reference electrode in $0.5 \mathrm{M} \mathrm{H}_{2} \mathrm{SO}_{4}$ containing $0.1 \mathrm{M} \mathrm{KCl}$ (at $100 \mathrm{mV} \mathrm{s}^{-1}$ scan rate), to obtained the cleaned SPCE surface. Then, the SPCE surface was treated electrochemically diazotized following the procedure described by Baranton and Belanger [11], with slight modification. The electrochemical modification of SPCE with an in-situ generated 4-carboxyphenyl diazonium salt (4-CPDS) and end group activation was carried out as described in our previous work [10]. For aptamer immobilization, the activated SPCE was incubated with $30 \mu \mathrm{L}$ of anti-mycotoxins or kanamycin aptamer solution at an optimized concentration (1 or $2 \mu \mathrm{M})$ for $4 \mathrm{~h}$ under humidity environment. After incubation, the electrode were incubated with $30 \mu \mathrm{L}$ of 1 $M$ ethanolamine solution (60 $\mathrm{min}$ ) to deactivate remaining active succinimide groups and block unreacted sites with prior rinsing with BB. After rinsing, the electrode was further incubated with $100 \mu \mathrm{L}$ of $1 \%$ BSA solution for 60 min to completely block the unbound sites of the SPCE surface. The aptamer modified SPCEs were used directly as aptasensor or stored dry at $4^{\circ} \mathrm{C}$ for several days without any decrease in activity.

ii. Covalent aptamer immobilization via diamines spacers functionalized SPCE The immobilization of MB tagged anti-OTA aptamer on the SPCE surface was performed by electrochemical grafting of diamine long spacer chains on electrode. Electrografting of spacers was carried out in $0.05 \mathrm{M}$ of NBu4BF $40.5 \mathrm{M}$ acetonitrile solution containing $6 \mathrm{mM} \mathrm{HMDA}$, or $6 \mathrm{mM} \mathrm{NH} 2-\mathrm{PEG}-\mathrm{NH}_{2}$ (Mw 3000 or Mw 10.000). Then cyclical cathodic potential was applied between $0.3 \mathrm{~V}$ to $1.7 \mathrm{~V}$ (vs. $\mathrm{Ag} / \mathrm{AgCl}$ ) at $0.5 \mathrm{Vs}^{-1}$, leading to the covalent attachment of spacers via an amine linkage. The modified electrodes were used directly for aptamer immobilization. The redox probe MB was tagged on $3^{\prime}$ of the aptamer whereas $5^{\prime}$ was modified with $\mathrm{COOH}$ group. The terminal carboxylic group of aptamer was activated using $100 \mathrm{mM}$ EDC and $25 \mathrm{mM}$ $\mathrm{NHS}$ in $0.1 \mathrm{M}$ MES buffer for $60 \mathrm{~min}$. After end group activation, $20 \mu \mathrm{L}$ of activated 
aptamer solution $(2 \mu \mathrm{M})$ were incubated onto the SPCE surface for 45 min under humid environment. The anti-OTA-aptamer modified SPCEs can be used directly for sensing or stored dry at $4^{\circ} \mathrm{C}$ for several days without any decrease in the sensitivity.

\section{c. Electrochemical measurements}

i. Electrochemical impedance measurements

All the electrochemical impedimetric experiments were carried out at an applied potential of $0.1 \mathrm{~V}(\mathrm{vs} . \mathrm{Ag} / \mathrm{AgCl}$ ) in a frequency range of $1 \mathrm{kHz}$ to $0.1 \mathrm{~Hz}$ at $\mathrm{AC}$ amplitude of $5 \mathrm{mV}$. Impedance data of SPCE electrodes were recorded after each successive modification steps. The impedance spectra were plotted in the form of complex plane diagrams (Nyquist plots, $-Z_{i}$ vs. $Z_{r}$ ) and fitted to a theoretical curve corresponding to an equivalent circuit (Randles Circuit) with FRA software. The parameter $R_{s}$ corresponds to the resistance of the solution, $R_{c t}$ is the charge transfer resistance between the solution and the electrode surface, $C_{D L}$ is associated with the double-layer capacitance and the Warburg parameter (W) corresponds to the diffusion of the redox probe. In order to compare the results obtained from different electrodes used and to obtain independent, reliable and reproducible results, relative and normalized signals were required. Accordingly, an analytical parameter, $\Delta_{\text {ratio, }}$ was defined using the $R_{c t}$ values in the following equations:

$$
\begin{gathered}
\Delta_{\text {ratio }}=\Delta_{s} / \Delta_{p} \\
\Delta_{s}=R_{\mathrm{ct}}(\text { Apt-analyte })-R_{\mathrm{ct}}(\text { bare electrode }) \\
\Delta_{\mathrm{p}}=\mathrm{R}_{\mathrm{ct}}(\text { aptamer })-\mathrm{R}_{\mathrm{ct}}(\text { (bare electrode })
\end{gathered}
$$

Where $R_{c t(A p t-a n a l y t e)}$ was the electron-transfer resistance measured after incubation with the target analyte, i.e. AFB1 or KANA, Rct(aptamer) was the electron-transfer resistance

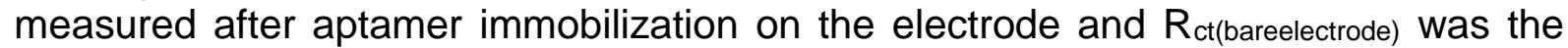
electron-transfer resistance of the bare electrode.

ii. Diferential Pulse Voltammetry mesuarements

After immobilization of aptamers and incubation of OTA on SPCE surface, DVP measurements were carried out using BB solution under the following conditions; modulation time, 0.02 interval time $0.5 \mathrm{~s}$, initial potential $0.4 \mathrm{~V}$, end potential $0.15 \mathrm{~V}$, step potential $0.005 \mathrm{~V}$, modulation amplitude $0.10005 \mathrm{~V}$, stand by potential $0 \mathrm{~V}$. Upon the oxidation of MB tagged on aptamers, the height of the resulting peak wave form was recorded and plotted against OTA concentration to construct a calibration curve.

\section{Results and discutions}

\section{a. Design strategy of electrochemical aptamer based sensor}

i. Reagentetless aptasensors with MB tagged aptamer

The aptamer immobilization via electrochemical oxidation of long chain HMDA or PEGs on the SPCE surface formed a cluster of long spacer arms on the transducer surface, leaving sufficient distance between the redox probe and the electrode surface. Consequently, in absence of analyte (OTA), the flexible conformation of aptamer enabled the electrical communication of MB with the electrode. After binding with analyte, the aptamer self-assembled into an antiparallel G-quadruplex structure, which electron transfer of MB from the electrode surface. Hence, the increasing concentration of analyte resulted in increase of electrochemical signal (Scheme 1). 


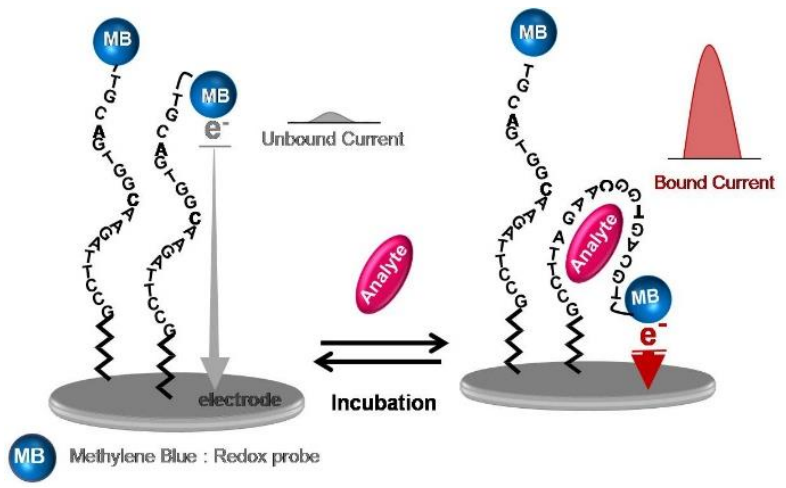

Scheme 1. Schematic representation of OTA biosensing principle using MB tagged aptamer

ii. Label free Impetimetric aptasensors

Impedimetric aptasensor strategy are based on the monitoring of changes in the electron transfer resistance at the electrode in the presence of as the redox active probe. The interaction between analyte and the aptamer induced an proportion increase of the electron transfer electrode depending on analyte concentration.

\section{b. Characterization of aptasensor using CV, FT-IR and EIS}

The electrochemical characterizations were performed in the presence of a relatively low concentration $(1 \mathrm{mM})$ of reversible redox couple $[\mathrm{Fe}(\mathrm{CN}) 6]^{4-/ 3-}$.

i. $\quad$ CV and FT-IR characterization

The change in peak-to-peak separation and peak current in voltammograms at different modification steps are corresponds to the electron transfer-rate constant and, thus the electron transfer resistance. The modification of electrode surface at different steps was characterized by a decrease in the current response and an increase in the peak-to-peak separation (Fig. 1A). A characteristic quasi-reversible redox peak with the cathodic and anodic current peak ratio of approximately one and the peak-to-peak separation of $1.1 \mathrm{~V}$ was obtained for bare SPCE. The large peak to-peak separation value $(E p)$ indicates the quasi reversible reaction behavior of the electrode surface. After the modification (4-CP/SPCE), the electron-transfer resistance between the redox probe and electrode surface was increased due to the formation of an organic layer with negatively charged carboxyl groups which could acts as an electrostatic barrier, and obviously the anodic and cathodic peaks have nearly disappeared in the CV (SPCE/4-CP). On aptamer immobilization, the peaks for the redox couple have decreased further due to the complex aptamer structure on the electrode surface. Finally, analyte (AFB1 or KANA) detection; a firm decrease in the current response is accompanied. Furthermore, the FT-IR spectrum confirmed the presence of absorption bands as expected for presence of free carboxylic group at $3440-2908 \mathrm{~cm}^{-1}$ with absorption bands at $1640 \mathrm{~cm}^{-1}$ (str., $-\mathrm{C}=\mathrm{O}$ of carboxyl) and out-of-plane bending at 915 $\mathrm{cm}^{-1}$ (oop bend., $-\mathrm{OH}$ of acid) for diazotized electrodes.

ii. Impedimetry (EIS) characterization

The successful modification of the functionalized layer was also confirmed through EIS measurements. The Nyquist plots consist of a semicircle portion and a linear portion, which semicircle portion at higher frequencies represents the electron-transfer resistance and the linear portion corresponds to the diffusion resistance is shown in the Fig. 1. As seen in Fig. 1B, the bare SPCE exhibited only a very small semicircle domain, implying a very low electron-transfer resistance, $R_{c t}$, for the redox probe (30 
$\left.\mathrm{k} . \mathrm{cm}^{-2}\right)$. After the 4-CP electro-grafting, the Rct tremendously increased $\left(2400 \mathrm{k} . \mathrm{cm}^{-2}\right)$, due to the negatively charged terminal carboxyl groups $\mathrm{COO}-$ which acting as an electrostatic barrier that repels the $\left[\mathrm{Fe}(\mathrm{CN})_{6}\right]^{4-3^{-}}$anions and inhibits the electrontransfer between the redox probe and the electrode. In the next step, the terminal carboxyl group activates the formation of ester and thus the $R_{c t}$ decreased considerably $\left(300{\mathrm{k} . \mathrm{cm}^{-2}}^{-2}\right.$ ) compared to the $R_{\mathrm{ct}}$ of $4-\mathrm{CP}$ modified electrode (2400 $\mathrm{k} . \mathrm{cm}^{-2}$ ). Immobilization of the aptamer induced an increase in the semi-circular

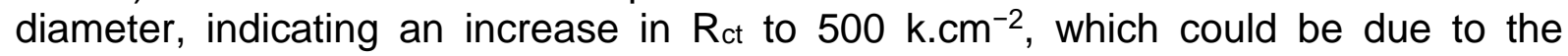
presence of phosphate groups of aptamer that repelled the negatively charged redox probes and thus increased the $R_{c t}$. Afterward, the $R_{c t}$ increased $\left(900 \mathrm{k} . \mathrm{cm}^{-2}\right)$ when the aptasensor was used to detect analyte. The increase in $R_{c t}$ could be partially due to the conformational changes of the immobilized aptamer upon target binding.
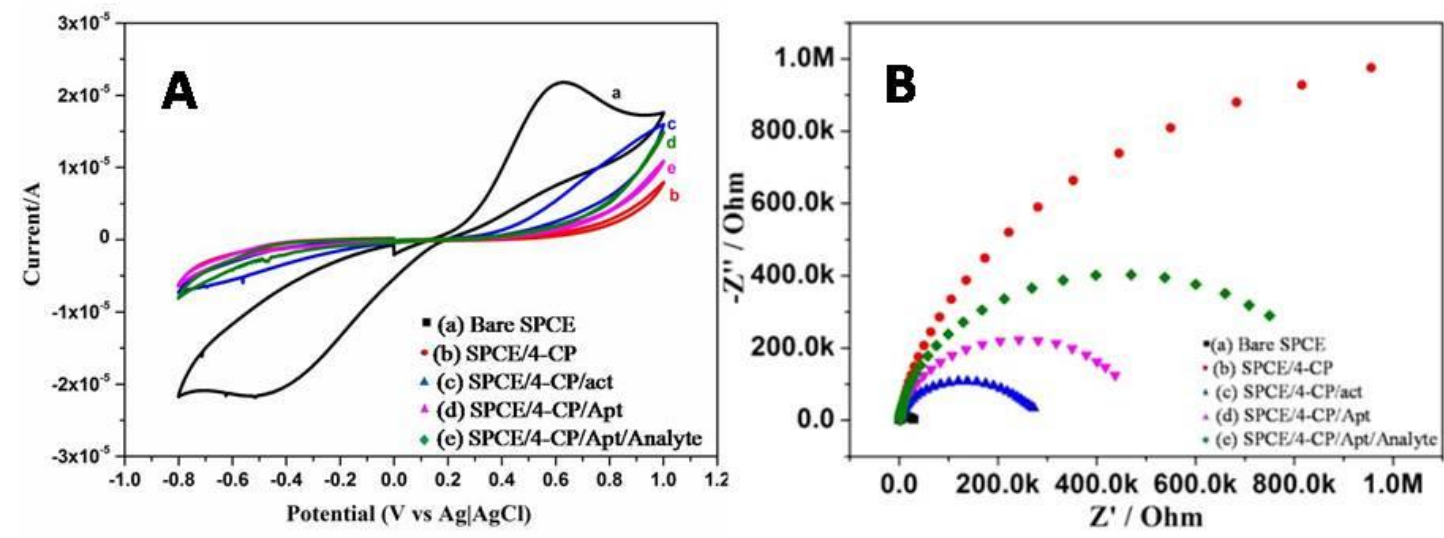

Fig 1. (A): Cyclic voltammograms and (B) Nyquist plots of the EIS analysis of $1 \mathrm{mM}$ $\left[\mathrm{Fe}(\mathrm{CN})_{6}\right]^{3-14-}$ at $100 \mathrm{mVs}^{-1}$ for (a) bare SPCE, (b) SPCE/4-CP electrode, (c) SPCE/4-CP/act electrode, (d) SPCE/4-CP/Apt electrode and (e) SPCE/4-CP/Apt/AFB1 electrode; at an applied potential of $0.1 \mathrm{~V}$ (vs. $\mathrm{Ag} / \mathrm{AgCl}$ ) using a frequency range of $1 \mathrm{KHz}$ to $0.1 \mathrm{~Hz}$ and an $\mathrm{AC}$ amplitude of $5 \mathrm{mV}$.

\section{c. Analytical performance of the aptasensors}

All the experimental variables such as $\mathrm{pH}$, temperature, aptamer concentration as well as the incubation time of aptasensor were optimized, which has direct or indirect effect on aptasensor performance.

\section{i. Ochratoxin detection via reagentless aptasensor}

In order to validate the feasibility of this new approach for the analysis of OTA, the quantitative determination of various OTA concentrations between $0.01-5 \mathrm{ng} \mathrm{mL}^{-1}$ was achieved by measuring the oxidation peak current of $\mathrm{MB}$ after background subtraction by DPV (Fig 2A). A calibration curve was performed after 60 min of incubation time of analyte. The standard curve was well described by a linear relationship (Fig 2B) with a linear regression equation $y=0.4035 x p=0.90311, r=0.9976$. The limit of detection (LOD) was calculated as the concentration of analyte corresponding to the 3 times "S/N" ratio. The calculated RSD value is $3.75 \%$ which represent the mean RSD obtained from five different OTA concentrations, where five different modified SPCE are used as replicates for each concentration. As result, rational engineering of the aptamer structure to create large conformation changes upon OTA binding leads to improved aptasensor performance for OTA detection presents high analytical performance in terms of linear range $\left(0.01-5 \mathrm{ng} \mathrm{ml}^{-1}\right)$, sensitivity $\left(0.4035 \mathrm{ng}^{-\mathrm{ml}^{-1}}\right)$, and

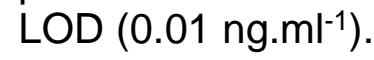




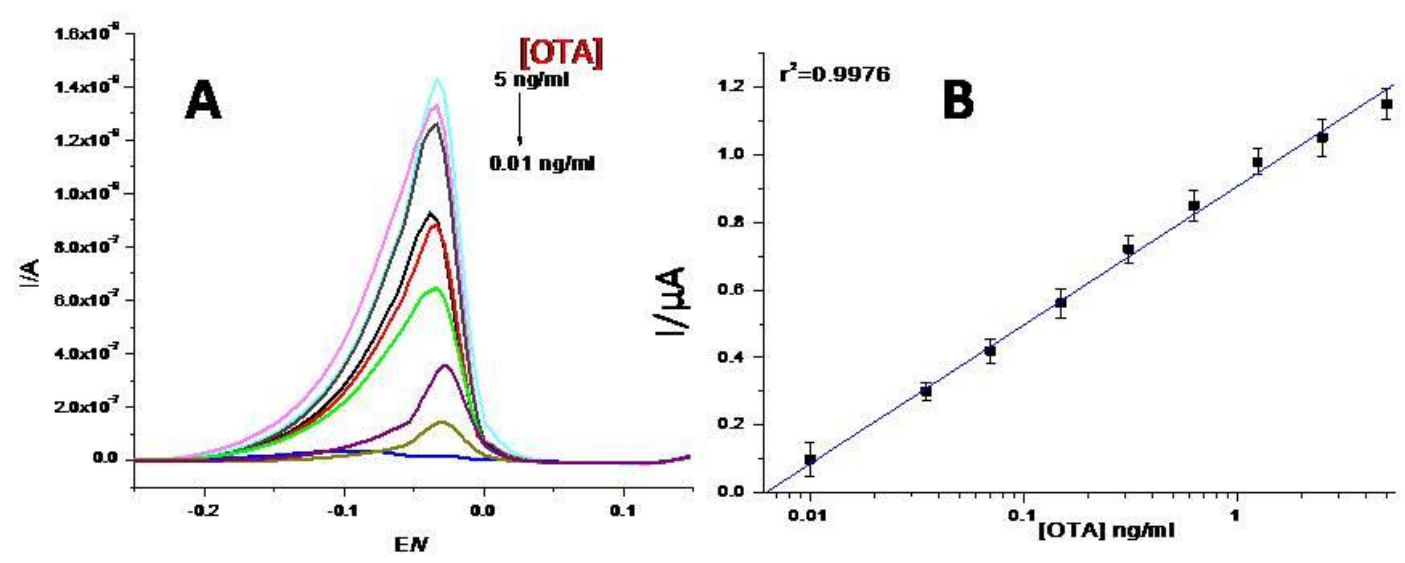

Fig 2 (A) Differential pulse voltamograms and (B) calibration curve obtained for different OTA concentrations ( 0.01 to $5 \mathrm{ng} \mathrm{ml}^{-1}$ ) using reagentless methylene blue aptasensors

ii. Aflatoxins and Kanamycin detection via label free aptasensors

The AFB1 and KANA was dispensed on the aptamer bound SPCE electrodes (SPCE/4-CP/Apt) at various concentrations between 0.125-16.0 $\mathrm{ng} \mathrm{mL}^{-1}$ and 2.5-300 ng. $\mathrm{mL}^{-1}$ respectively for AFB1 and KANA, with an incubation time of $1 \mathrm{~h}$. After the incubation, the impedimetric measurements were recorded and obtained results are shown in Fig. 3 . The corresponding $R_{c t}$ values were calculated and presented as $\Delta_{\text {ratio }}$ against AFB1 and KANA concentration in the graph. It can be seen from the figure 3 A that the $\Delta_{\text {ratio }}\left(R_{\mathrm{ct}}\right)$ values of the aptasensor increased with the rise in the concentrations of AFB1, and the obtained linear region, $0.125-2.0 \mathrm{ng} \mathrm{mL}^{-1}$, has been presented in the inset (Fig. 3B), with a good determination range from $0.125-16.0 \mathrm{ng}$ $\mathrm{mL}^{-1}$. The LOD and correlation coefficient values are $0.125 \mathrm{ng} \mathrm{mL}^{-1}$ and 0.99 . The obtained cross reactivity responses of aptasensors were in the range of less than $10 \%$ against other structural and non structural analogues concerning antimicrobial residues and mycotoxins. The obtained results showed in the $\Delta$ ratio response were due to the interaction of target analyte with aptasensor and developed no cross reactivity against other analogues. The precision studies (intra and inter day) showed the high repeatability of developed platform with a maximum standard deviation of 4.69 $\%(n=3)$. Analytical performance of the two label free aptasensors for AFB1 and KANA compared with existing results detection is compared in terms of LOD, linear range and recovery values (92-100.6\%). As the results showed, both aptasensors are capable of detecting analyte with high sensitivity and reproducibility, meeting the requirement of regulatory standard i.e. $150 \mathrm{ng} \cdot \mathrm{mL}^{-1}$. A LOD was found to be 0.15 ng. $\mathrm{mL}^{-1}$. 


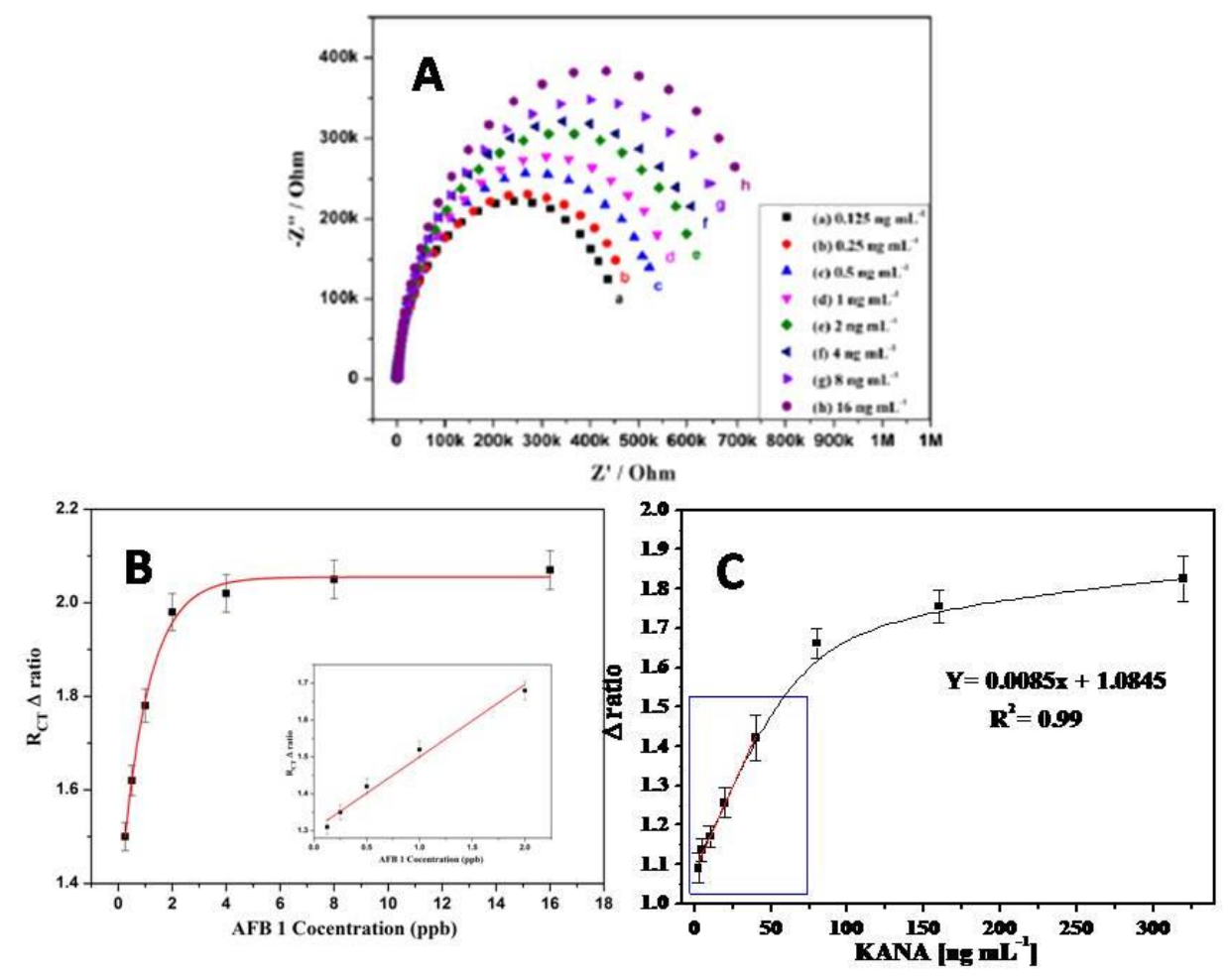

Figure 3 (A) Nyquist plots of anti-AFB1 aptamer modified SPCEs with different concentrations (ng mL-1) of AFB1; (a) 0.125, (b) 0.25, (c) 0.5, (d) 1.0, (e) 2.0, (f) 4.0, (g) 8.0 and (h)16.0; (B) The calibration curve corresponding to the detection of AFB1 based on change in electron-transfer resistance (RCT), which is presented as $\triangle$ ratio; The inset represents the linear region of the calibration curve of the aptasensor. $(C)$ The calibration curve corresponding to the detection of KANA.

\section{Conclusions}

In summary, we have developed the electrochemical aptasensor for the detection of mycotoxins and antibiotic residue. The proposed prototypes of developed aptasensors offers the advantages of disposability, label free and portability due to the implication of SPCEs system using diazonium coupling, which proves its feasibility for on-site analysis. The utility of EIS and DPV techniques demonstrates a sensitive method to quantify the change of electrode surface during aptasensor fabrication. To the best of our knowledge, these are among the few reports in the implication of SPCEs functionalized EIS-aptasensor for detection of kanamycin and ochratoxin A. The precision studies proves that developed aptasensors exhibits the good repeatability and reproducibility. On basis of the obtained results, it can be envisaged that the developed platforms could be further extended for detection of contaminants in other food matrices.

\section{Acknowledgement}

Atul Sharma (A.S.) would like to thanks EUPHARTES (ERASMUS Mundus) Doctoral Fellowship program.

\section{References}

[1] Boonen, J.; Malysheva, S.V.; Taevernier, L.; Diana Di Mavungu, J.; De Saeger, S.; De Spiegeleer, B., (2012), Human skin penetration of selected model mycotoxins, Toxicology, 301, 21-32. 
[2] Guo, X.; Wen, F.; Zheng, N.; Luo, Q.; Wang, H.; Wang, H.; Li, S.; Wang, J., (2014), Development of an ultrasensitive aptasensor for the detection of aflatoxin B1, Biosensors and Bioelectronics, 56, 340-344.

[3] Cabello, F.C., (2006), Heavy use of prophylactic antibiotics in aquaculture: a growing problem for human and animal health and for the environment, Environmental Microbiology, 8(7), 1137-1144.

[4] Leung, K.-H., He, H.-Z., Chan, D.S.-H., Fu, W.-C., Leung, C.-H., Ma, D.-L., (2013), An oligonucleotide-based switch-on luminescent probe for the detection of kanamycin in aqueous solution, Sensors and Actuators B: Chemical, 177, 487-492.

[5] Commission regulation (EC) No. 1881/2006 of 19 December 2006 setting maximum levels for certain contaminants in foodstuffs," Official Journal of the European Union, (2006), L364/5L364/24.

[6] Commission Regulation (EC) No. 401/2006 laying down the methods of sampling and analysis for the official control of the levels of mycotoxins in foodstuffs, Official Journal of the European Union, vol. L70, (2006) 1234.

[7] Codex Alimentarious Commission, Joint FAO/WHO Food Standards Programme "Report of The Fourteenth Session of The Codex Committee on Residues of Veterinary Drugs In Foods" 2003, Cl 2003/11-Rvdf.

[8] Food Safety and Standard Authority of India (FSSAI) "Manual of Methods for analysis of Foods - Antibiotics and Hormone Residue" Lab Manual 2012, 15, 1-57.

[9] Homem V., Santos L., (2011), Degradation and removal methods of antibiotics from aqueous matrices-A review, Journal of Environmental Management, 92(10), 2304-2347.

[10] Hayat, A., Barthelmebs, L., Marty, J.-L., (2012), Electrochemical impedimetric immunosensor for the detection of okadaic acid in mussel sample, Sensors and Actuators B: Chemical, 171-172, 810-815.

[11] Baranton, S., Belanger, D., (2005), Electrochemical derivatization of carbon surface by reduction of in situ generated diazonium cations. The Journal of Physical Chemistry B 109(51), 24401-24410. 